\section{An Analysis of Vehicle-to-Infrastructure Communications for Non-Signalised Intersection Control under Mixed Driving Behaviour}

\author{
G. Budan, K. Hayatleh, D. Morrey, P. Ball, P. Shadbolt
}

\begin{abstract}
Intersection control has an important role in the management of urban traffic to ensure safety, high traffic flow and to prevent congestion. Recently, a growing body of literature has been reported on the theme of non-signalised intersection control in which traffic lights are replaced with intelligent road side units. Data from several studies suggest that non-signalised control could reduce vehicle delays and fuel consumption significantly whilst ensuring safety. However, there is little published data on the impact of the mixed driving behaviour with human-driven vehicles and Autonomous Vehicles (AV). This paper investigates the emerging role of connectivity and vehicle autonomy in the context of traffic control under the mixed driving behaviour scenario. The concepts of Vehicle-to-Infrastructure (V2I) communications and Multi-Agent Systems (MAS) are central to achieving a robust and reliable traffic-light-free intersection control. Comprehensive computer simulation results on a four-way intersection indicate over 96\% reduced average vehicle delay and $37 \%$ less fuel consumption with the nonsignalised control solution compared to the traffic light control. The outcome of this study offers some important insights into enabling cooperation between vehicles and traffic infrastructure via V2I communications, in order to make more efficient real-time decisions about traffic conditions, whilst ensuring a higher degree of safety.
\end{abstract}

Keywords V2I, Connected Vehicle, Autonomous Vehicle, Non-Signalised Intersection, Traffic Simulation, VISSIM

\section{Introduction}

A traffic intersection can be seen as a shared resource where exclusive access is given for a limited time duration. Traffic light control provides uniform information to all users to signal intersection crossing and this is one of the main reasons for the success of traffic light control to date [1]. Signalised control systems generally use fixed-position road sensors such as loop detectors, radars and cameras to determine the current traffic state and to optimise the signal parameters. Therefore, the efficiency of the signalised control depends on the number of sensors deployed [2]. Furthermore, the proportion of fatalities in road accidents that occurred at intersections was between 34-38\% in the United Kingdom (UK) and 20-21\% across the European Union (EU) throughout the years 20052014 [3].

It is expected that Connected Autonomous Vehicles (CAV) will reduce traffic accidents significantly as great majority of accidents happen due to human error [4]. CAVs are also believed to reduce fuel consumption and traffic congestion mainly due to increased sensory precision and the smoother acceleration and speed control capabilities of these vehicles which, in turn, enables more efficient usage of existing road networks [5]. The aforementioned advantages of CAVs compared to connected human-driven vehicles (CHV) are not only due to autonomy, but connectivity will also play a key role to enable cooperation between vehicles and traffic infrastructure via V2I communications [6]. Considering that there will be a long transitional period in which traditional human-driven vehicles and CAVs will co-exist in traffic [7], it is essential to accommodate mixed driving behaviour while creating non-signalised intersection control systems.

One study by [1] draws our attention to the technology path dependency for intersection control, and the authors argue that the existing traffic control methods should not form the basis when considering a next generation disruptive technology such as non-signalised intersection control with CAVs. The aim of an intersection control system with Connected Vehicles $(\mathrm{CV})$, is to achieve a high degree of cooperation between its users, which will lead to a mutually beneficial outcome that reduces the number of accidents and provides lower vehicle delay [8].

In this paper, we implement four different CAV driving behaviours, similar to those proposed by [9], by modifying the vehicle dynamics such as speed profiles, lane positioning, vehicle headways and gap acceptance parameters in order to analyse the road capacity, safety, carbon emissions and vehicle delays under non-signalised intersection control. CAVs are assumed to be level 5 autonomous as defined by [10]. The main contributions of this paper are as follows. First, we build a comprehensive microscopic traffic simulation environment in PTV VISSIM [11], to analyse the mixed driving behaviour impacts on the traffic flow at intersections, and the mutual interaction of these vehicles. Second, we implement $\mathrm{CAV}$ driving behaviours, coded in $\mathrm{C}++$, from cautious to aggressive that represents user preference or car manufacturer choice. Third, we implement a non-signalised intersection control method based on resolving vehicle trajectory conflicts and spatio-temporal reservation of the intersection crossing area, and we compare this against traffic light control through simulation work.

The remainder of this paper is divided into four sections. The next section gives the related work on intersection control under mixed driving behaviour. In section 3, we will then outline the methodology for intersection control system modelling. Section 4 explains the simulation set-up and presents the findings of the research, focusing on the mixed driving behaviour under various traffic demand levels. Section 5 concludes the paper and gives the future work.

\section{Background}

Intersection control without traffic lights can be categorised as centralised and decentralised based on the decision making strategy. In centralised control, there is at least one decision that is made for all vehicles at an intersection by a central controller with V2I communication capabilities. On the other hand, in decentralised control, all decision making is done by the approaching vehicles by utilising Vehicle-to-Vehicle (V2V) communications [5]. In this paper, non-signalised intersection control will refer to centralised control.

The existing literature on intersection control for connected vehicles is extensive and focuses particularly on signalised control. However, there is a relatively small body of literature that is concerned with traffic light-free intersection control. 
Considering that all levels of vehicle automation will be present in traffic for a foreseeable future, level 0 and level 5 vehicles are integrated into intersection control by [12] and [13] and a First-Come-First-Served (FCFS) vehicle scheduling based control system is proposed in which existing traffic light infrastructure is still used only for human drivers to signal right-of-way. However, CAVs can still traverse the intersection on a red light if the centralised controller confirms a conflict-free reservation. The simulation results indicate a significant increase in average vehicle delay as the percentage of level 0 vehicles increase. This issue is addressed by [14] by integrating all autonomy levels of vehicles. A constrainedbased reservation control system is proposed that takes the autonomy level of a vehicle into account in order to generate individual vehicle trajectories and speed profiles. Similarly, a priority-based control system with the objectives of speed maximisation and idle time reduction is proposed by [15].

Another study by [16] draws our attention to the feasibility issues of vehicle scheduling methods for level 0 vehicles, and the authors propose a sequence-based protocol in which human drivers are informed via in-vehicle display whether or not they are authorized to cross the intersection. In a followup study [17], the aforementioned sequence-based protocol is extended to a high-level signalisation system in which platooning is encouraged for vehicle group crossing. Other methods in the literature for non-signalised intersection control under mixed driving behaviour include Mixed Observability Markov Decision Process (MOMDP) [18], multi-objective evolutionary algorithm [19], Model Predictive Control (MPC) [7] and optimisation formulation [20].

\section{System Model}

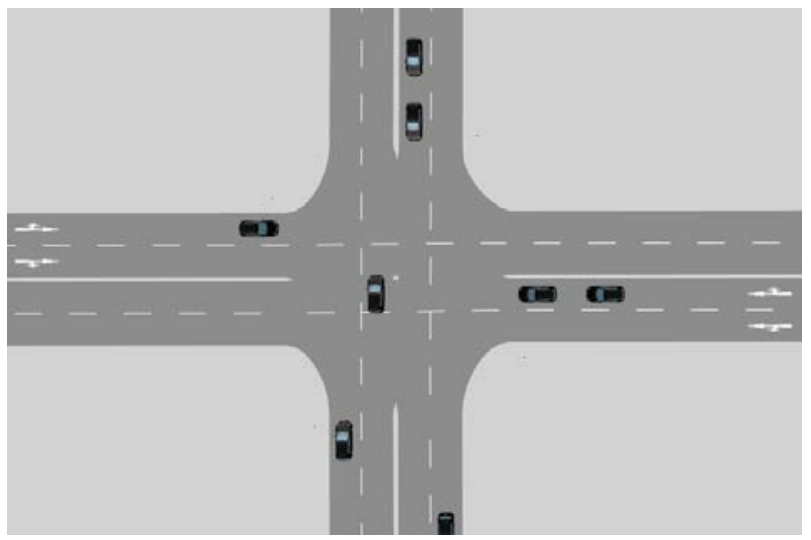

Fig. 1 Intersection layout with four approaching links and two lanes on each link

We consider the problem of vehicle coordination at a nonsignalised intersection with four approaching links and two lanes on each link as shown in Fig. 1. Left and right turn movements are allowed in addition to through movement and left-hand traffic is considered in this study. Denoting $N$ as the total number of approach lanes, we define the intersection area where incoming lanes $l_{1} . . l_{n} \in N$ intersect as a Critical Area (CrA) that has the potential for lateral vehicle collision and consists of Conflict Points (CPs).
Cooperative centralised intersection control is realised by information exchange i.e. positions, dynamics, attributes etc. between the Intersection Control Agent (ICA) and road Vehicle Agents (VA). The data is transmitted periodically as part of the facilities layer within the Intelligent Transport Systems (ITS) architecture [21] and it is called Cooperative Awareness Message (CAM). ITS G5 control channel is used to disseminate CAM in a single hop communication. Therefore, direct communication range is required to exchange CAM data. CAM generation frequency range for VAs is specified as between $10 \mathrm{~Hz}(100 \mathrm{~ms})$ and $1 \mathrm{~Hz}(1 \mathrm{sec})$ whereas it can be greater than $1 \mathrm{~Hz}$ for road infrastructures such as an ICA [22]. In this study, the data frequency is set as $10 \mathrm{~Hz}$ for vehicles and $0.5 \mathrm{~Hz}$ for ICA.

The sequence diagram is given in Fig. 2 for the V2I communications between the VAs and ICA. First, VAs plan their intersection crossing trajectory including the arrival/exit lanes, the arrival time to the $\mathrm{CrA}$ and the crossing speed profile when they are within the control range of the intersection. Second, VAs transmit the data set, which is summarised in Table 1 below, to the ICA in order to reserve a crossing time window.

Table 1 Vehicular data set transmitted by VAs to the ICA

\begin{tabular}{ll}
\hline VA Data & Description \\
\hline veh_id & Unique vehicle identification number \\
msg_id & Message identification number \\
ica_id & ICA identification number \\
veh_type & Vehicle type including the autonomy level \\
dist_to_cra & Distance to the intersection critical area \\
veh_v & Current vehicle speed \\
veh_a & Current vehicle acceleration/deceleration \\
veh_length & Vehicle length \\
veh_width & Vehicle width \\
veh_t_arr & Estimated arrival time to the critical area \\
veh_lane_arr & Intersection arrival lane \\
veh_lane_exit & Intersection exit lane \\
veh_v_cross & Vehicle target crossing speed \\
\hline
\end{tabular}

As the approaching VAs request for intersection crossing, the received data is buffered on the ICA side and processed at every control interval. Vehicle scheduling consists of two main stages; vehicle sequencing and conflict resolution. Vehicle sequencing is the stage in which unordered list of approaching vehicles are ordered and prioritised based on the intersection control objective. In this study, we extend the First-Come-First-Served (FCFS) sequencing protocol which was introduced by [23]. Our extension includes a strategy for vehicles with updated approach plan (i.e. late arrival time). For example, when a VA requests a change of reservation due to later arrival time than originally estimated, the ICA evaluates the new arrival time. If trajectory conflicts exist, then the ICA cancels the original crossing time window and pushes the subject VA at the end of the crossing queue instead of shifting the crossing time windows of all the other VAs that are scheduled to cross after the subject VA. The rationale of this strategy is to keep the reservation changes to a minimum which is especially important for CHVs.

Following this, VAs can request for an earlier crossing time if they are at full stop at the entrance of the intersection waiting for their original crossing time. This enables VAs to utilise any 
cancelled crossing time windows due to an updated approach plan as explained previously and helps reduce incurred vehicle delays.

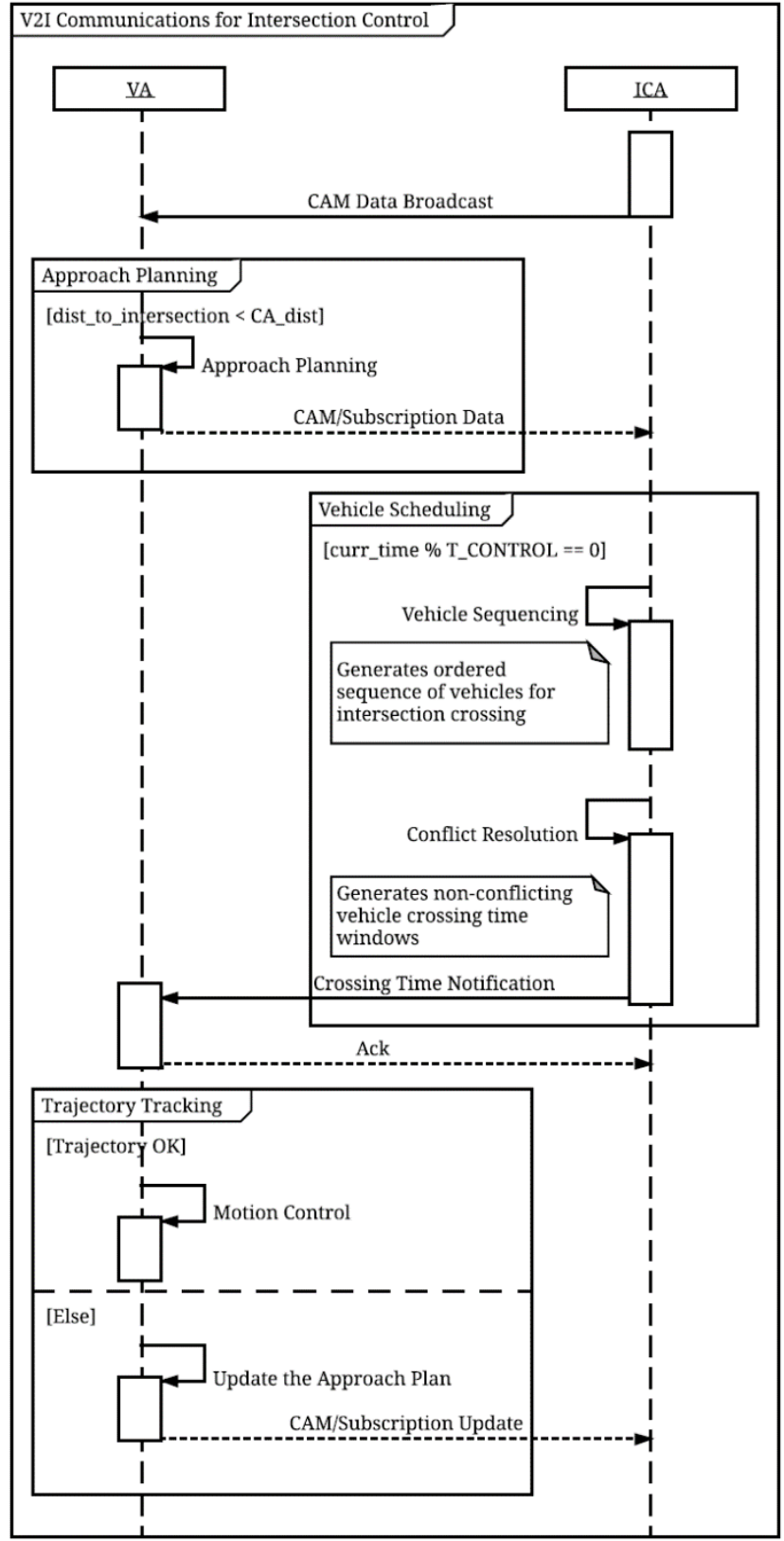

Fig. 2 Sequence diagram for the V2I communications between ICA and VA

In conflict resolution stage, the CPs for vehicles with conflicting trajectories are identified and crossing time windows are generated for each vehicle. Assigned priorities in the previous stage are used to determine which conflicting vehicle to be allocated an earlier reservation. $\mathrm{CP}$ management helps to reduce the complexity of reservation-based control system [6] compared to the method proposed by [22] that discretizes the whole intersection $\mathrm{CrA}$ into a grid of tiles. Our objective in this stage is to generate vehicle crossing time windows in order to avoid collisions. To this end, we implemented different driving behaviour parameters for level 0 and level 5 vehicles which will be explained in the next section.

We consider the following assumptions in the control system. Vehicles in traffic are all connected and a mixture of level 0 and 5 vehicles. Level 0 vehicles are under the control of a human driver at all times whereas level 5 vehicles perform all aspects of the dynamic driving tasks without any human intervention [10]. Human drivers obey the crossing time windows allocated to them, and therefore, they do not try to traverse the intersection without holding a valid reservation. Vehicles are not allowed to reverse, overtake or change lane inside the control area of the intersection. Vehicles are equipped with Global Positioning System (GPS) sensors and disseminate their location with measurement accuracy of no worse than 1 metre. Vulnerable Road Users (VRU) such as pedestrians, cyclists and motorcyclists are not considered. Driving behaviour outside the intersection control area is beyond the scope of this work.

\section{Performance Evaluation}

\subsection{Driving Behaviour}

The default driving behaviours in VISSIM are based on Wiedemann car following models [24]. The users can adjust the parameters of these models in order to create different levels of CAV behaviours as proposed by [9]. However, this approach has a major limitation in terms of implementing a non-signalised intersection control strategy, as this requires real-time interaction with the vehicles. For that reason, the Wiedemann driver model in VISSIM is replaced with an external version which is implemented as a Dynamic Link Library (DLL) file in the programming language $\mathrm{C}++$. During a simulation run, the DLL file is called every $100 \mathrm{~ms}$ for each vehicle in the network to control the behaviour in the next time step based on the intersection control strategy.

In this study, $4 \mathrm{CAV}$ level 5 driving behaviours $\{C A V$ $B 1 \ldots C A V B 4\}$ are implemented and the associated parameter values are summarised in Table 2 . The interested readers can refer to [24] and [9] for a more detailed explanation of the parameters. These parameters are determined to vary the driving behaviour from cautious to aggressive, similar to [9].

\subsection{Simulation Model}

The block diagram of the simulation model is shown in Fig. 3. A modular design approach is taken in order to facilitate integration of different algorithms for driving behaviours and vehicle scheduling strategies. Traffic network and road layout are created in VISSIM. The Component Object Model (COM) interface gives access to traffic data and functions contained in VISSIM during a simulation run. National Instruments (NI) Laboratory Virtual Instrument Engineering Workbench (LabVIEW) platform is used in this study as a central control hub that provides test automation, data visualisation, V2I communications and intersection control functions. 


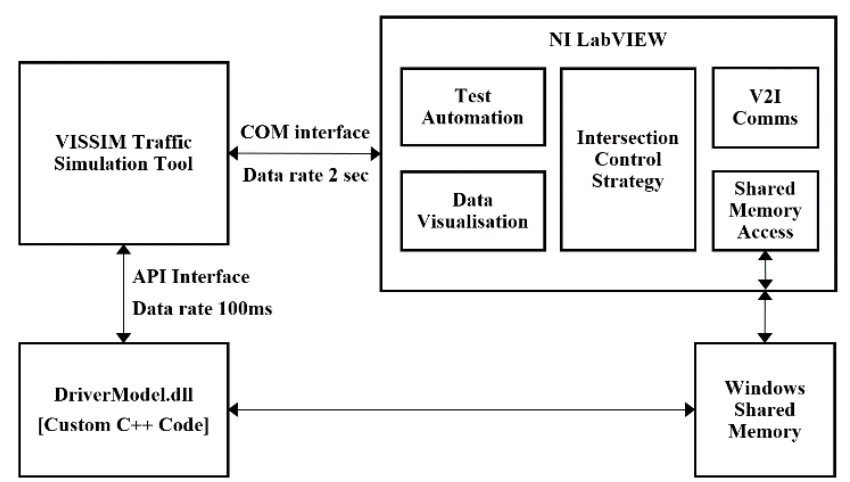

Fig. 3 Block diagram of the mixed driving behaviour traffic simulation platform

Table 2 Driving behaviour parameters for CHVs and CAVs

\begin{tabular}{|c|c|c|c|c|c|c|}
\hline Parameter & Description & $\mathrm{CHV}$ & CAV B1 & CAV B2 & CAV B3 & CAV B4 \\
\hline $\mathrm{CCO}$ & Desired standstill distance between vehicles [m] & 1.5 & 1.0 & 0.9 & 0.6 & 0.5 \\
\hline $\mathrm{CC} 1$ & Headway time from the vehicle in front [sec] & 0.9 & 0.8 & 0.7 & 0.6 & 0.5 \\
\hline $\mathrm{CC} 2$ & Headway longitudinal distance oscillation [m] & 4.0 & 0.0 & 0.0 & 0.0 & 0.0 \\
\hline $\mathrm{CC} 3$ & Time to recognise a preceding slower vehicle $[\mathrm{s}]$ & 8.0 & 8.0 & 8.0 & 8.0 & 8.0 \\
\hline $\mathrm{CC} 4$ & Negative desired speed difference $[\mathrm{m} / \mathrm{s}]$ & 0.35 & 0.05 & 0.05 & 0.05 & 0.05 \\
\hline $\mathrm{CC} 5$ & Positive desired speed difference $[\mathrm{m} / \mathrm{s}]$ & 0.35 & 0.05 & 0.05 & 0.05 & 0.05 \\
\hline CC6 & Influence of vehicle distance on speed oscillation & 0.0 & 0.0 & 0.0 & 0.0 & 0.0 \\
\hline $\mathrm{CC} 7$ & Oscillation during acceleration $[\mathrm{m} / \mathrm{s} 2]$ & 0.25 & 0.30 & 0.35 & 0.40 & 0.45 \\
\hline $\mathrm{CC} 8$ & Acceleration when starting from standstill $[\mathrm{m} / \mathrm{s} 2]$ & 3.5 & 3.6 & 3.7 & 3.8 & 3.9 \\
\hline CC9 & Acceleration at $80 \mathrm{kph}[\mathrm{m} / \mathrm{s} 2]$ & 1.5 & 1.6 & 1.7 & 1.8 & 1.9 \\
\hline
\end{tabular}

The driving behaviours are implemented external to VISSIM and the data exchange is established via the Application Programming Interface (API). V2I communications are implemented through Windows Shared Memory which enables bi-directional data exchange for multi-threaded applications.

To evaluate the system performance, each driving behaviour was tested under 4 different traffic flow conditions, ranging from 500 to 3000 vehicles per hour (veh/h). In this study, low traffic and high traffic conditions are defined as traffic flow under $1000 \mathrm{veh} / \mathrm{h}$ and over $1000 \mathrm{veh} / \mathrm{h}$ respectively. Mixed traffic scenarios were created at varying penetration rates of CAVs, ranging from $10 \%$ to $90 \%$. With an 18000 second simulation period, 400 simulation cases were executed in total. Each scenario was repeated 5 times with a different random seed and the data was obtained by averaging the results of the multiple simulation runs. The overall performance under non-signalised intersection control was compared against the stage-based fixed-time traffic light control. Traffic light signal phase and timing parameters were optimised for each scenario with the built-in optimisation tool in VISSIM that takes the traffic flow and demand ratio conditions into account.

\subsection{Metrics}

In order to provide a quantitative evaluation of the intersection control system, four types of metrics were selected in this study: 1) average vehicle delay; 2) average vehicle speed; 3 ) average queue length; and 4) average fuel consumption. Together, these metrics indicate the mobility and sustainability measures of the control system.

\subsection{Experimental Results}

The average vehicle delay gain with the non-signalised control under varying traffic flow conditions is shown in Fig. 4. It can be seen from this chart that vehicle delay gain is significant, between $96-100 \%$ under low traffic. What stands out in the chart is that traffic light control starts to perform better as traffic flow increases which is also supported by [25]. Closer inspection of the data shows that CAV penetration rate also has a positive effect on the vehicle delay. Although performing worse than traffic light at $10 \%$ CAV penetration rate, it still reduces the vehicle delay by $36 \%$ compared to traffic with only human drivers.

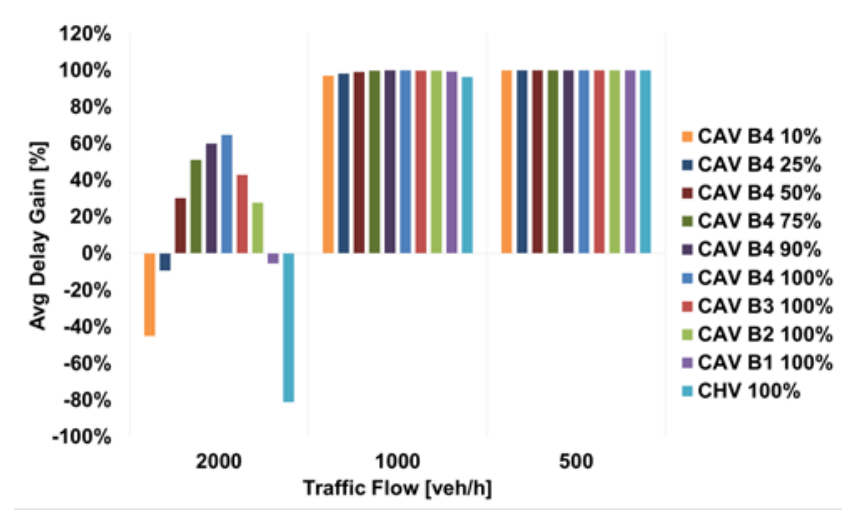

Fig. 4 Percentage of average vehicle delay gain under varying traffic flow conditions

The average vehicle speed gain under varying traffic flow conditions is shown in Fig. 5. The average speed with nonsignalised control is $18-25 \%$ less than traffic light control under low traffic flow. This is mainly due to the fact that vehicles have allocated time windows to cross the intersection. Hence, the vehicle speed is set in a way to arrive to the intersection at the reserved time. From this data, we can also see that average speed is improved under high traffic flow, reaching $27 \%$ for $\mathrm{CHVs}$. 


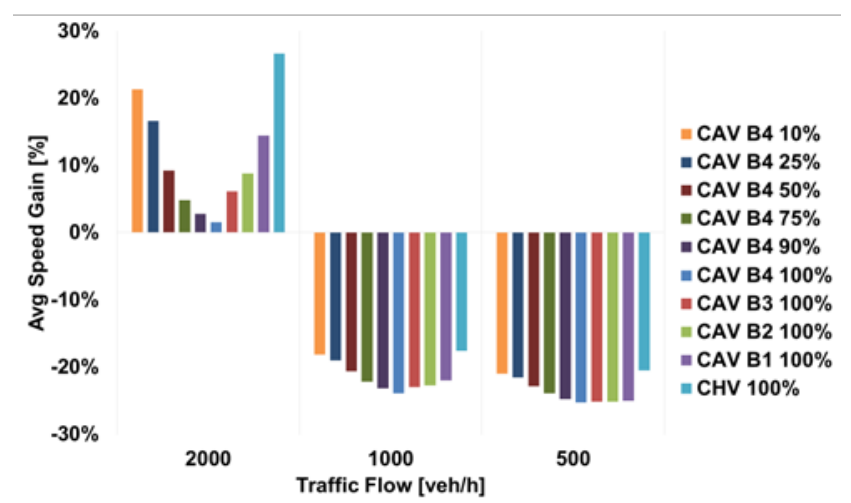

Fig. 5 Percentage of average vehicle speed gain under varying traffic flow conditions

The average queue length gain under varying traffic flow conditions is shown in Fig. 6. Similar to vehicle delay gain, the average queue length improvements are between $93-100 \%$ under low traffic. However, the queue length gets more than doubled for $\mathrm{CHV}$ s under high traffic. The data also shows that minimum of $75 \% \mathrm{CAV}$ penetration is required in order to get any queue length improvements under high traffic compared to traffic light control.

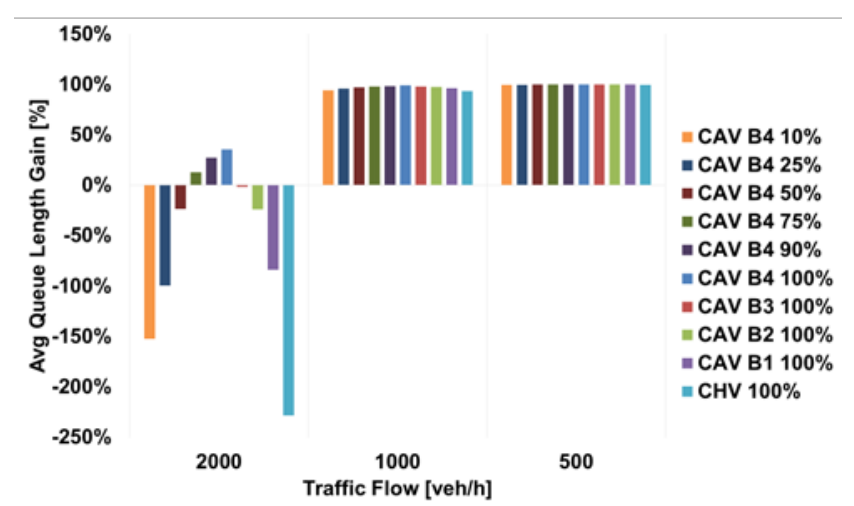

Fig. 6 Percentage of average queue length gain under varying traffic flow conditions

The average fuel consumption gain under varying traffic flow conditions is shown in Fig. 7. What is striking about the data on this figure is that cautious driving behaviour (CAV B1) gives the highest fuel consumption improvements, reaching up to $42 \%$ which is followed by other driving behaviours between $38-40 \%$ under low traffic. The greatest fuel consumption improvement under high traffic is again observed with CAV B1 driving behaviour. In fact, all CAV behaviours benefit from fuel consumption savings under high traffic as opposed to $\mathrm{CHV}$ which is $23 \%$ worse than traffic light control under high traffic.

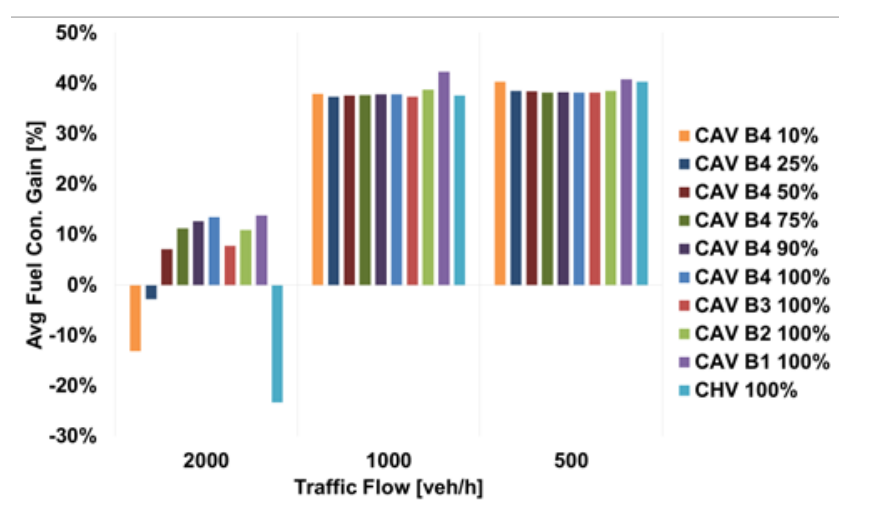

Fig. 7 Percentage of average fuel consumption gain under varying traffic flow conditions

\section{Conclusion}

This paper examined the emerging role of connectivity in the context of non-signalised intersection control and created a state-of-the-art computer simulation framework to validate the proposed non-signalised intersection control method. The performance of the non-signalised control was compared against the traffic light control in a four-way intersection network that was created in VISSIM traffic simulation tool under various CAV driving behaviour models. In summary, the implementation results show that FCFS-based vehicle scheduling method offers $42 \%$ less average fuel consumption, over $96 \%$ reduced average vehicle delays and 93\% less average queue length under low traffic conditions compared to the traffic light control. However, traffic light control starts to outperform FCFS-based non-signalised control as traffic congestion increases. Further research is required in order to explore more advanced vehicle scheduling methods that give better performance in terms of the metrics used in this study under all traffic flow conditions. A future study investigating machine learning methods such as reinforcement learning and neural networks for vehicle crossing order generation would be very interesting. Taken together, the results of this study offer some important insights into enabling cooperation between vehicles and traffic infrastructure via V2I communications, in order to make more efficient real-time decisions about traffic conditions.

\section{References}

1. Mladenovic, Abbas, Blyth, P.-L., \& Kosonen, I. (2016). Intersecting our mobilities: path dependence from manuallyoperated semaphore to self-driving vehicles? In 2016 IEEE International Symposium on Technology and Society (ISTAS) (pp. 1-6). IEEE. doi:10.1109/ISTAS.2016.7764042

2. Atkins. (2016). Research on the impacts of connected and autonomous vehicles (CAVs) on traffic flow Stage 1: Evidence Review. Retrieved from

https:/www.gov.uk/government/uploads/system/uploads/attach ment_data/file/530091/impacts-of-connected-and-autonomousvehicles-on-traffic-flow-summary-report.pdf

3. European Commission. (2016). Traffic Safety Basic Facts on Junctions. European Commission, Directorate General for Transport. Retrieved from https://ec.europa.eu/transport/road_safety/sites/roadsafety/files/ $\mathrm{pdf} /$ statistics/dacota/bfs2016_junctions.pdf

4. Fagnant, D. J., \& Kockelman, K. (2015). Preparing a nation for autonomous vehicles: opportunities, barriers and policy recommendations. Transportation Research Part A: Policy and Practice, 77, 167-181. doi:10.1016/j.tra.2015.04.003

5. Rios-Torres, J., \& Malikopoulos, A. A. (2016). A Survey on the Coordination of Connected and Automated Vehicles at Intersections and Merging at Highway On-Ramps. IEEE Transactions on Intelligent Transportation Systems, 1-12. doi:10.1109/TITS.2016.2600504

6. Chen, L., \& Englund, C. (2016). Cooperative Intersection Management: A Survey. IEEE Transactions on Intelligent Transportation Systems, 17(2), 570-586. doi:10.1109/TITS.2015.2471812

7. Liu, X., Ma, K., \& Kumar, P. R. (2015). Towards provably safe mixed transportation systems with human-driven and automated vehicles. In 2015 54th IEEE Conference on Decision and 
Control (CDC) (pp. 4688-4694). Osaka, Japan: IEEE. doi:10.1109/CDC.2015.7402950

8. Mladenovic, M. N., Abbas, M., \& McPherson, T. (2014). Development of socially sustainable traffic-control principles for self-driving vehicles: The ethics of anthropocentric design. In 2014 IEEE International Symposium on Ethics in Science, Technology and Engineering (pp. 1-8). IEEE. doi:10.1109/ETHICS.2014.6893448

9. Atkins. (2016). Research on the impacts of connected and autonomous vehicles (CAVS) on traffic flow Stage 2: Traffic Modelling and Analysis Technical Report. Retrieved from https:/www.gov.uk/government/uploads/system/uploads/attach ment_data/file/530091/impacts-of-connected-and-autonomousvehicles-on-traffic-flow-summary-report.pdf

10. SAE On-Road Automated Vehicle Standards Committee. (2014). Taxonomy and Definitions for Terms Related to OnRoad Motor Vehicle Automated Driving Systems. SAE Standard J3016. SAE International. doi:10.4271/J3016_201401

11. PTV. (2016). PTV VISSIM 9 User Manual. Karlsruhe, Germany: PTV AG.

12. Dresner, K., \& Stone, P. (2006). Making autonomous intersection management backwards-compatible. In A. Cohn (Ed.), AAAI'06 proceedings of the 21st national conference on Artificial intelligence (Vol. 2, pp. 1865-1866). Boston, Massachusetts: AAAI Press. Retrieved from http://www.aaai.org/Papers/AAAI/2006/AAAI06-311.pdf

13. Sharon, G., \& Stone, P. (2017). A Protocol for Mixed Autonomous and Human-Operated Vehicles at Intersections. In In proceedings of The 2nd International Workshop on Agentbased modeling of urban systems (ABMUS 2017). Sao Paulo, Brazil. Retrieved from http://www.cs.utexas.edu/ aim/papers/ABMUS17-Sharon.pdf

14. Au, T.-C., Zhang, S., \& Stone, P. (2015). Autonomous Intersection Management for Semi-Autonomous Vehicles. In D. Teodorović (Ed.), The Routledge Handbook of Transportation. Abingdon: Routledge. doi:10.4324/9781315756684.ch7

15. Qian, X., Gregoire, J., Moutarde, F., \& De La Fortelle, A. (2014). Priority-based coordination of autonomous and legacy vehicles at intersection. In 17th International IEEE Conference on Intelligent Transportation Systems (ITSC) (pp. 1166-1171). Qingdao, China: IEEE. doi:10.1109/ITSC.2014.6957845

16. Perronnet, F., Abbas-Turki, A., Buisson, J., El Moudni, A., Zeo, R., \& Ahmane, M. (2012). Cooperative intersection management: Real implementation and feasibility study of a sequence based protocol for urban applications. In 2012 15th International IEEE Conference on Intelligent Transportation Systems (pp. 42-47). Anchorage, Alaska, USA: IEEE. doi:10.1109/ITSC.2012.6338769

17. Perronnet, F., Abbas-Turki, A., \& El Moudni, A. (2013). A sequenced-based protocol to manage autonomous vehicles at isolated intersections. In 16th International IEEE Conference on Intelligent Transportation Systems (ITSC 2013) (pp. 18111816). The Hague, The Netherlands: IEEE. doi:10.1109/ITSC.2013.6728491

18. Sezer, V., Bandyopadhyay, T., Rus, D., Frazzoli, E., \& Hsu, D. (2015). Towards autonomous navigation of unsignalized intersections under uncertainty of human driver intent. In 2015 IEEE/RSJ International Conference on Intelligent Robots and Systems (IROS) (pp. 3578-3585). Hamburg, Germany: IEEE. doi:10.1109/IROS.2015.7353877

19. Onieva, E., Hernandez-Jayo, U., Osaba, E., Perallos, A., \& Zhang, X. (2015). A multi-objective evolutionary algorithm for the tuning of fuzzy rule bases for uncoordinated intersections in autonomous driving. Information Sciences, 321, 14-30. doi:10.1016/j.ins.2015.05.036

20. Zhao, L., Malikopoulos, A., \& Rios-Torres, J. (2017). Optimal Control of Connected and Automated Vehicles at Roundabouts: An Investigation in a Mixed-Traffic Environment. arXiv preprint. Retrieved from http://arxiv.org/abs/1710.11295

21. ETSI. (2010). ETSI EN 302665 Intelligent Transport Systems (ITS); Communications Architecture. ETSI (Vol. 1). ETSI. Retrieved from http://www.etsi.org/deliver/etsi_en/302600_302699/302665/01. 01.01_60/en_302665v010101p.pdf

22. ETSI. (2014). ETSI EN 302 637-2 V1.3.2 Intelligent Transport Systems (ITS); Vehicular Communications; Basic Set of Applications; Part 2: Specification of Cooperative Awareness Basic Service. ETSI (Vol. 1.3.2). ETSI. Retrieved from http://www.etsi.org/deliver/etsi_en/302600_302699/30263702/0 1.03.02_60/en_30263702v010302p.pdf

23. Dresner, K., \& Stone, P. (2004). Multiagent Traffic Management: A Reservation-Based Intersection Control Mechanism. In AAMAS '04 Proceedings of the Third International Joint Conference on Autonomous Agents and Multiagent Systems - Volume 2 (pp. 530-537). Washington: IEEE Computer Society. doi:10.1109/AAMAS.2004.190

24. Wiedemann, R., \& Reiter, U. (1992). Microscopic traffic simulation: the simulation system MISSION, background and actual state. Project ICARUS (V1052) Final Report, 2, 1-53.

25. Levin, M. W., Boyles, S. D., \& Patel, R. (2016). Paradoxes of reservation-based intersection controls in traffic networks. Transportation Research Part A: Policy and Practice, 90, 1425. doi:10.1016/j.tra.2016.05.013 